\section{POST-HIERARCHICAL RACE:}

Reconsidering the Nature of

Hierarchy within Haslanger's

Account of Race

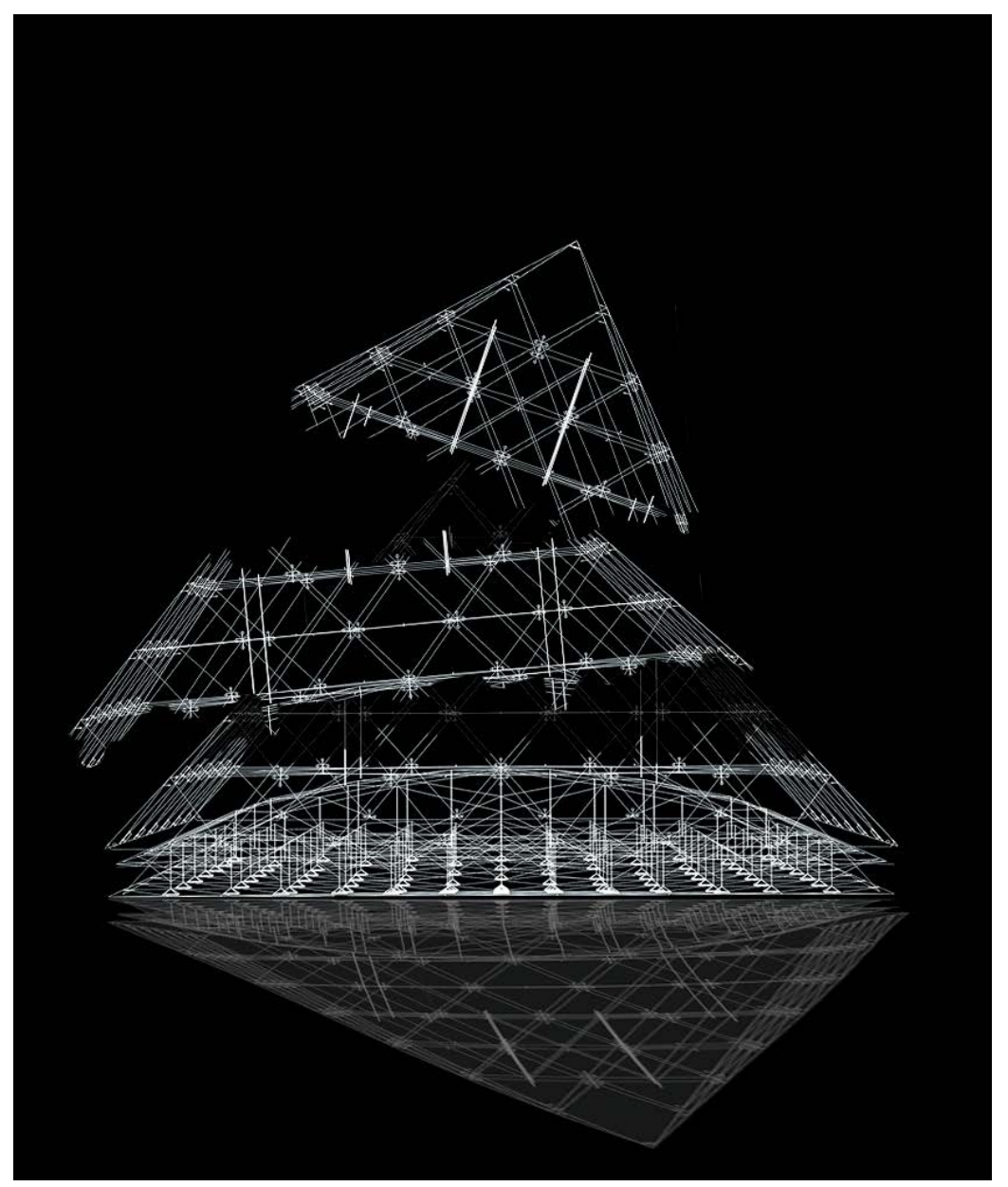

MAX DAVIDSON-SMITH

\section{ABSTRACT}

In this essay, I consider Sally Haslanger's social constructivist account of race and propose a modification to the nature of hierarchy specified. According to Haslanger, race will cease to exist post-hierarchy, given that she builds in a requirement of synchronic hierarchy for the existence of race. While Haslanger maintains that racial identity would linger beyond hierarchical treatment in the form of ethnicity, I will suggest this fails to provide adequate conceptual justice for the cultures and aesthetics which emerged out of past oppression. In response, I propose a modification which would allow us to recognize the possibility of post-hierarchical races. 


\section{INTRODUCTION}

Sally Haslanger's work has made a profound impact on the field of critical race theory. ${ }^{1}$ Her approach challenges any suggestion that there is a biological basis for race; instead, she focuses on our social ontology and the operation of race in everyday discourse. While broadly in agreement, I propose a modification to the nature of hierarchy within Haslanger's social constructivist account of race. I first outline Haslanger's social constructivist framework, defending her revisionary or "ameliorative" antiracist intentions, before focusing on her account of race as hierarchical social positioning. I then distinguish between Haslanger's requirement for synchronic ongoing hierarchy and a historical connection to hierarchy which is no longer occurring, before proposing to modify her account to allow both as constituting races within her social constructivist framework.

Justifying this modification, I outline two purposes for posthierarchical race as predicated on historical connection to hierarchy. First, post-hierarchical races maintain awareness of past hierarchical treatment in society and guard against its re-emergence. Secondly, the Black Art Movement will signify a positive case for introducing post-hierarchical races based on historical connection to hierarchy, which allows us to properly respect and also celebrate the cultures and aesthetics which emerged out of past oppression.

\section{SOCIAL CONSTRUCTIVISM AS A FRAMEWORK}

Haslanger makes use of the externalist referential account of meaning. Through locating what best explains our use of the term "race," she attempts to specify the nature of this concept. Given "ordinary folk" manage to talk about race independently of authority, this is an important consideration for Haslanger in the sense that everyday discourse largely accounts for the meaning of race in society. Resisting the need to defer to scientific experts, Haslanger is unashamedly engaged in the task of determining the meaning and nature of race as it is actively used in society. While genetics and biology suggest that there is no adequate biological referent, a social kind best refers to our ordinary usage of race. ${ }^{2}$ Despite race being socially constructed, its existence is as real as other ontological kinds.

Haslanger's framework also contains a distinctly ameliorative project with her account designed to provide "effective tools in the fight against injustice."3 Drawing on Hilary Putnam's semantic

1 Sally Haslanger, Resisting Reality: Social Construction and Social Critique (Oxford: Oxford University Press, 2012).

2 Haslanger, Resisting Reality, 307

3 Haslanger, Resisting Reality, 226 externalism, Haslanger argues the meaning of race is partially fixed by external factors. ${ }^{4}$ Race exists as a socially constructed kind which depends on our creation and usage in discourse for its maintenance. The fact that race as a concept is socially constructed makes society somewhat responsible in defining the concept for our legitimate purposes and therefore justifies the revisions conducted by Haslanger. In light of this, Haslanger asks what, if anything, can our concept of race do for us going forward. Against the objection that this approach distorts the ordinary meaning of race, Haslanger claims that, providing central functions of the term remain the same, and the revisionary goals being served are politically acceptable, her targeted concept will "represent itself as providing a ... (possibly revisionary) account of the everyday concepts." ${ }_{5}$ Motivated by a critical and antiracist goal, Haslanger's intentions are arguably justifiable and commendable.

\section{SOCIAL POSITIONING ACCOUNT OF RACE}

Haslanger's account of race examines how groups are socially positioned and what physical markers serve as a supposed basis for hierarchical treatment

A group is racialized iff its members are socially positioned as subordinate or privileged along some dimension (economic, political legal, social, etc.), and the group is "marked" as a target for this treatment by observed or imagined bodily features presumed to be evidence of ancestral links to a certain geographical region. ${ }^{6}$

Bodily features, called "color," are physical characteristics presumed to be linked to and explained by geography, such as hair texture or skin tone. According to Haslanger's account, "race is the social meaning of color," which positions members in a social hierarchy through systematic subordination or privilege. ${ }^{7}$ Race, therefore, functions through identification of individuals within and by a society based on a perception of physical features being linked to a purported geographical ancestry, thereby marking them out as a group for systematic hierarchical treatment.

By building hierarchy into an account of race, Haslanger intends to "locate the mechanisms of injustice and the levers for social change" central to her ameliorative antiracist intentions. ${ }^{8}$ Focusing specifically on the way in which hierarchy functions within race as a defining

\footnotetext{
4 Hilary Putnam, "The Meaning of 'Meaning,"' Minnesota Studies in the Philosophy of Science 7 (1975): 131-93, https://hdl.handle.net/11299/185225.

5 Haslanger, Resisting Reality, 224.

6 Hastar

Haslanger, Resisting Reality, 236.

Haslanger, Resisting Reality, 236

Haslanger, Resisting Reality, 184
} 
feature, Haslanger hopes individuals will realize their own position of privilege or subordination before considering their wider role in challenging and dismantling racial injustice.

\section{MODIFYING HASLANGER'S NATURE OF HIERARCHY}

I now propose a modification to the nature of hierarchy specified by Haslanger, where synchronic and ongoing color-based subordination or privilege is deemed necessary for the existence of races as social kinds. It is worthwhile to consider the possibility of a future without the existence of such color-based hierarchical treatment and subsequent implications for the existence of races. Accordingly, in a post-hierarchical future without color-based hierarchy, Haslanger maintains that races will cease to exist. This follows from the particular nature of hierarchy outlined within her account of race. ${ }^{9}$ Theodore Bach, however, suggests it is plausible that races might survive the loss of any hierarchical properties yet still linger in existence. ${ }^{10}$ While synchronic hierarchy seems to be an integral feature for the emergence of races, this does not mean ongoing hierarchy is the only means by which race might continue to exist. I propose Haslanger's account of hierarchy be modified to also include a historical connection to hierarchy as a constitutive factor for races to remain in existence. A post-hierarchical race would preserve some of the valuable features of a previously racialized group but within a societal context free from hierarchical treatment.

A key reason in favor of accounting for post-hierarchical race is that this plausibly captures our use of the concept, reflecting how we view races as capable of enduring rather than liable to simply disappear. Our intuitions here are being tested on what is currently only a hypothetical future. It does, however, seem that the concept of race has the potential for having more permanence than surviving based only on the contingency of hierarchical treatment. Even if we accept that synchronic hierarchical treatment is an important component of the concept of race and accounts for how it first arises, this does not mean that, were the conditions of hierarchy to change, race would necessarily be defunct. It is possible that, were the hierarchical circumstances to be eliminated, the concept of race might still hold some positive relevance in society.

The maintenance of races post-hierarchy might consist of members considered by themselves as well as by society as being meaningfully

9 Haslanger, Resisting Reality, 248-52.

10 Theodore Bach, "Review of Sally Haslanger, Resisting Reality: Social

Construction and Social Critique," Ethics 124, no. 3 (2014): 617, 10.1086/674829. historically connected to, but not currently experiencing, the past hierarchical treatment of racial ancestors.

[Post-hierarchical races definition]: A group (2) is racialized posthierarchy iff its members are positioned by themselves and adequately recognized by society as being meaningfully linked and historically or culturally connected to ancestors of a group (1), where that group (1) experienced hierarchical treatment on the basis of observed or imagined bodily features, "color" presumed to be evidence of ancestral links to a certain geographical region.

The proposed definition of post-hierarchical races should be considered as an addition but not a replacement to Haslanger's account, which is useful in allowing us to determine the continued existence of races post-hierarchy. When considering the initial emergence of races and their existence during synchronic hierarchy, Haslanger's original definition will be relevant.

On my modification, I have purposefully built in as a requirement that members of a post-hierarchical race play some part in deliberately and actively linking themselves with the former hierarchically-treated racial group. In doing so, we can encapsulate the fact that there is some degree of agency needed for post-hierarchical races to continue. I consider that this conceptual requirement may be useful in ensuring that the purpose of post-hierarchical race is beneficial. Given that there would not be post-hierarchical races lingering without intent and desire from the members of that group, we can reasonably infer that their reason for identifying and connecting with former racial groups serves a meaningful purpose and that what is being remembered and celebrated is likely to be worthwhile.

I have provided just a sketch of the nature of post-hierarchical race. In doing so, I have suggested post-hierarchical race would entail a collection of individuals who have chosen to closely associate themselves with a formerly hierarchically-treated racial group.

Importantly, this is based on a meaningful connection which might, for example, include following unique traditions or sustaining memories through the retelling of stories. Finally, in order for post-hierarchical race to be a useful feature of mutual cultural understanding, this connection to former racialized groups is adequately recognized as such by other members of society outside of these groups.

I will now make use of Haslanger's own ameliorative framework to justify my modification to the nature of hierarchy and the conceptual inclusion of post-hierarchical race. In doing so, we can suggest how post-hierarchical race might in fact better serve our legitimate political purposes. First, maintaining use of post-hierarchical race is an effective 
tool in guarding against the potential re-emergence of racial injustice. There is reason to suggest that by acknowledging the existence of posthierarchical race, which would be predicated only on past hierarchy, we are able to more effectively guard against the active re-emergence of that same past hierarchy or one of a similar kind. Introducing post-hierarchical race to our concepts can be of instrumental value in tackling the ever-present risk of hierarchical treatment from being a resurgent force in society, in both recognized and new forms. Over and above the transfer of memories and stories, post-hierarchical races apply to a real existing group which can help form a stronger and more effective reminder in the present of the reality of past oppression.

Ron Mallon detects an objection that maintaining the use of race connected to past hierarchy might be misunderstood as legitimizing or unnecessarily prolonging past oppression. ${ }^{11}$ While I agree that historical connection to hierarchy does keep past hierarchy alive, importantly, this is through remembrance instead of material practices. Rather than prolonging oppression, post-hierarchical race can spread awareness of past oppression, providing a much-needed guard against any reemergence of potentially dormant material hierarchy.

By way of rejoinder, it could be contested that remembrance might in fact lead to outlets for material practices of hierarchical treatment, perhaps by giving those inclined a central focus to rally against. Any empirical evidence that remembrance might encourage and enable hierarchical treatment is hard to locate; however, I suggest that if there is such a causal relationship, then it is only something which takes places in the exception. This is not to dismiss the importance of addressing any amount of material hierarchical treatment. It may be the case that remembrance really does, on occasion, risk the remnants of former hierarchy resurfacing, but this, in fact, enables us to confront the challenge head on. Through the often-public practice of remembrance, we can locate and address any such material hierarchical tendencies rather than have them perpetuated out of sight and away from the mainstream. Remembrance can help to tackle and confront hierarchical prejudices before they have the opportunity to spread further into society. It would be naive to assume that once hierarchical races have been dismantled, the synchronic hierarchy which led to their creation might never re-emerge. Haslanger's own ameliorative goal of tackling racial injustice is perhaps therefore better served by modifying race to include historical connection to hierarchy as constituting posthierarchical races.

11 Ron Mallon, "Race': Normative, Not Metaphysical or Semantic," Ethics 116 no. 3 (2006): 549, 10.1086/500495.
A further legitimate purpose for the modified account of hierarchy is that post-hierarchical race is intrinsically valuable in itself. With reference to the Black Art Movement, a positive case for race predicated on a historical connection to hierarchy can be established. Black aesthetics emerged during the Black Art Movement as a response to racial subordination during the 1960s. John Coltrane's composition, "Alabama," written in reaction to the 1963 Birmingham church bombing, provided a model for the emerging Black Consciousness Movement.

Trane was the spirit of the 60's

He was Malcolm X in New Super Bop Fire

Baaahhhh

Wheeeeee ... Black Art!!!'2

In this poem, Amiri Bakara, considered by many as a leader within the movement, points to Coltrane's significance in expressing a positive "Black" racial identity. ${ }^{13}$ Born out of hierarchical subordination and acute forms of oppression, black aesthetics established a positive reclamation of race both by and for that group. It is possible to point to other cases where a new aesthetic or culture was in part crystallized by the hierarchical treatment of a racial group. Richard Courage provides one such study of the impact of black artists during the depression era saying, "Chicago's black artists of the 1930s and 1940s sought to reframe perceptions of African American life and to re-present the black subject as something other than an object of contempt or amusement beneath the privileged gaze of the white viewer." 14 If we were to fast-forward to a post-hierarchical future, Haslanger's requirement of synchronic hierarchy becomes problematic as it means that black aesthetics will no longer refer to a real group given that, for Haslanger, the race has ceased to exist. But even if-as we undoubtedly strive for-synchronic hierarchy ceased, black aesthetics, which emerged in response to previous hierarchical treatment and are intimately bound up with a racial group, would still be relevant to our society.

Haslanger's account has two problematic implications. First, there is a concern about aesthetic appreciation. If the representational content of art in some way impacts its aesthetic value, then by rejecting

12 Amiri Bakara, AM/TRAK (New York: Nadja Editions, 1979), st. 5, http:// eclipsearchive.org/projects/AMTRAK/html/contents.html.

13 Bakara, AM/TRAK

14 Richard A. Courage, "Re-Presenting Racial Reality: Chicago's New (Media) Negro Artists of the Depression Era," Technoetic Arts: A Journal of Speculative Research 11, no. 2-3 (2012): 317, 10.1386/tear.10.2-3.309_1. 
the existence of post-hierarchical race, Haslanger in effect reduces the value of such art by denying its applicability to any real existing group. The content might tell a story, but an aspect of its meaning and reality is taken away by its failure to properly refer to race. Following on, it also seems that for those individuals who do strongly self-associate with past hierarchical racial groups, perhaps by celebrating the distinct culture and aesthetic which emerged, Haslanger's claim that races do not exist post-hierarchy implies that these individuals are in some sense misguided. Their opinion would be wrong in that while they can perhaps celebrate the Black Art Movement, they are mistaken to think of themselves as belonging to that same group.

By contrast, within my modified account, allowing for posthierarchical races means that those groups, historically connected with past subordination, can preserve what emerged as a positive culture and a celebrated aesthetic by tying this to a real, continuing, and existing race. Haslanger might object that my modification is merely semantic given that what I am talking about is just racial identity, which can be adequately captured by the term "ethnicity." 15 For Haslanger, the concept of race is something which we should aim to ultimately eliminate, seeing as it is not a meaningful concept without a colorbased hierarchy. She suggests there are other terms instead, including ethnicity, which could be used as a way of recognizing and addressing past injustices. However, it is not clear, even as an idealized concept, how the concept of ethnicity might operate in a post-hierarchical context, leaving some doubt about its utility and value in this situation. Furthermore, Haslanger's decision to do away with race seems to rest on the imperative that "the main issue is how we draw distinctions between humans for the purposes of justice." ${ }^{16}$ Her preoccupation with dismantling contemporary racial injustice is commendable but falls short of acknowledging the full potential for what our concept of race might be able to achieve. Specifically, by doing away with the present reality of race in a post-hierarchical age, the concept of ethnicity does not adequately provide justice to the cultures which emerged out of historical racial subordination. If we accept that providing such conceptual justice and recognition is a legitimate political purpose, then we are encouraged instead to modify our account of race to serve this goal as well as tackling synchronic injustices.

\section{CONCLUSION}

Having outlined Haslanger's ameliorative social constructivist framework, I have proposed modifying the synchronic nature of hierarchy within her account of race. This includes historical connection to hierarchy as a constitutive factor for the existence of post-hierarchical races. My modification serves two main purposes which justify maintaining the use of race post-hierarchy. First, serving Haslanger's own antiracist goal, historical connection to hierarchy is one tool which helps guard against the re-emergence of synchronic hierarchy. In addition, post-hierarchical race can provide conceptual justice to the cultures and aesthetics formed out of historical racial oppression which remain important to recognize in their own right. Haslanger's framework provides the opportunity to formulate a more complete account of race containing both aspects of hierarchy, therefore best tackling racial injustice while preserving the posterity of racial cultures. 


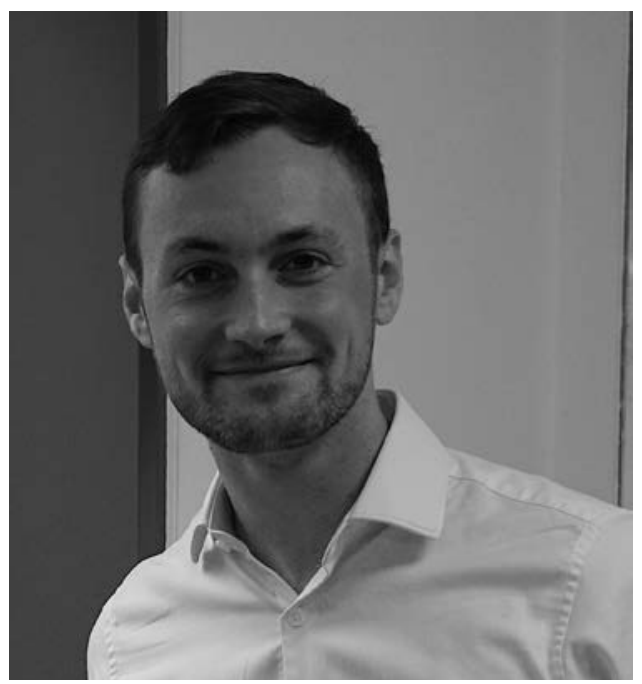

Max Davidson-Smith is a final-year student at the University of Leeds,

Yorkshire, England, where he is studying

philosophy and politics. His academic

interests lie in political philosophy and

theory as well as metaphysics. In addition,

Max is keen to explore ethical and

aesthetical discussions around artificial

intelligence and technology. 